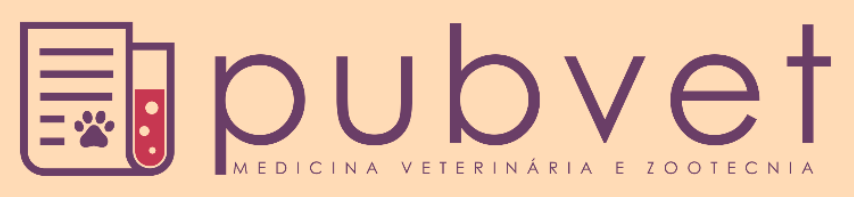

ISSN 1982-1263

https://doi.org/10.31533/pubvet.v12n9a175.1-8

\title{
Avaliação das alterações de exames bioquímicos indicativos de função renal e hepática em cães seniors e geriátricos
}

\author{
Amanda Finsterbuch ${ }^{1 *}$, Carlos Eduardo Nogueira Martins $^{2} \bullet$, Francielle D. de \\ Medeiros $^{3}{ }^{\bullet}$, Michele Muller Fialkowski ${ }^{\oplus}$, Pricilla Pozzatti ${ }^{5}$
}

${ }^{I}$ Acadêmica de medicina veterinária no Instituto Federal Catarinense, Araquari- SC Brasil.

${ }^{2} Z$ Zotecnista, Dr, Docente do Curso de Medicina Veterinária, Instituto Federal Catarinense, Araquari- SC.

${ }^{3}$ Médica Veterinária, Esp, Laboratório de Análises Clínicas MediVet, Joinville-SC.

${ }^{4}$ Médica Veterinária, Esp, Badanievet Serviços em Patologia Clínica Veterinária, Joinville- SC.

${ }^{5} \mathrm{MSc}$, Docente do Curso de Medicina Veterinária, Instituto Federal Catarinense, Araquari-SC. Orientador. E-mail: pricilla.pozzatti@ifc.edu.br

*Autor para correspondência, E-mail: amanda.finsterbuch@yahoo.com.br

\begin{abstract}
RESUMO. A população de animais geriatras vem crescendo consideravelmente nos últimos anos, consequentemente, o número de atendimentos na rotina clínica veterinária destes animais também veio a demonstrar um forte aumento. Como uma ferramenta útil no diagnóstico de enfermidades ou até mesmo para o monitoramento da saúde animal, as análises sanguíneas têm como objetivo identificar alterações que possam ser reflexo de algum processo patológico. Dentro das análises sanguíneas, o perfil bioquímico, se interpretado de forma correta, concedem referências quanto ao estado clínico de um animal, bem como seu equilíbrio nutricional, status imunológico, acompanhamento de tratamentos e prognósticos. Devido a importância dos parâmetros bioquímicos dentro do diagnóstico de enfermidades, principalmente na população geriatra, o objetivo deste estudo foi avaliar a ocorrência das alterações de exames bioquímicos que indicam modificações nas funções renal e hepática de cães idosos e classificá-las em relação ao sexo e idade. Para isto, foram coletados laudos de exames bioquímicos realizados em dois laboratórios do município e efetuadas triagens quanto a idade dos animais, seguida das triagens referentes as alterações hepáticas e renais e outra para sexo. A alteração com maior frequência na população de cães geriatras e seniors revelou- se ser Fosfatase Alcalina com 50,8\% (2.206/4.341) e $41,6 \%$ (979/2.352) respectivamente. O segundo analito com a maior frequência de alterações foi Ureia com 44,2\% (1.923/4.341) em cães geriatras e 29,9\% (705/2.352) em cães seniors. A relevância do sexo com relação a idade se mostrou significativa nos marcadores Fosfatase Alcalina e Ureia.
\end{abstract}

Palavras chave: alanina aminotransferase, cão, creatinina, diagnóstico, fosfatase alcalina, idosos, ureia

\section{Assessment of alterations in biochemical exams indicative of renal and hepatic function concerning sex and in elderly and geriatric dogs - a retrospective study between 2015 and 2017 in Joinville - SC}

\footnotetext{
ABSTRACT. The population of geriatric animals has grown considerably in the last years, consequently, the number of visits in the veterinary clinical routine of these animals also showed a strong increase. As a useful tool in the diagnosis of diseases or even for the monitoring of animal health, blood tests are aimed at identifying changes that may be a reflection of some pathological process. Within the blood analyzes, the biochemical profile, if correctly interpreted, gives references regarding the clinical state of an animal, as well as its nutritional balance, immunological status, follow-up of treatments and prognoses. Due
} 
to the importance of biochemical parameters in the diagnosis of diseases, especially in the geriatric population, the objective of this study was to evaluate the occurrence of alterations in biochemical tests that indicate changes in the renal and hepatic functions of elderly dogs and to classify them in relation to gender and age. For this, biochemical exams were collected in two laboratories in the city and the age of the animals was screened, followed by screenings for liver and kidney alterations and another for sex. The most frequent alteration in the population of geriatric and senior dogs was Alkaline Phosphatase with $50.8 \%$ (2.206/ 4.341) and 41.6\% (979/2.352), respectively. The second analyte with the highest frequency of changes was Urea with $44.2 \%(1,923 / 4,341)$ in geriatric dogs and $29.9 \%(705 / 2.352)$ in senior dogs. The relevance of sex in relation to age was significant in Alkaline Phosphatase and Urea markers.

Keywords: alanine aminotransferase, dog, creatinine, diagnosis, alkaline phosphatase, elderly, urea

\section{Evaluación de las alteraciones de exámenes bioquímicos indicativos de función renal y hepática en perros adultos y geriátricos - estudio retrospectivo entre 2015 y 2017 en Joinville - SC}

RESUMEN. La población de animales geriátricos ha crecido considerablemente en los últimos años, por lo que el número de consultas en la rutina clínica veterinaria de estos animales también ha demostrado un fuerte aumento. Como una herramienta útil en el diagnóstico de enfermedades o incluso para el monitoreo de la salud animal, los análisis sanguíneos tienen como objetivo identificar alteraciones que puedan ser reflejo de algún proceso patológico. En los análisis sanguíneos, el perfil bioquímico, si se interpreta correctamente, concede referencias en cuanto al estado clínico de un animal, así como su equilibrio nutricional, estado inmunológico, seguimiento de tratamientos y pronósticos. Debido a la importancia de los parámetros bioquímicos dentro del diagnóstico de enfermedades, principalmente en la población geriátrica, el objetivo de este estudio fue evaluar la incidencia de las alteraciones de exámenes bioquímicos que indican modificaciones en las funciones renal y hepática de perros longevos y clasificarlas en relación al sexo y edad. Para ello, fueron recolectados laudos de exámenes bioquímicos realizados en dos laboratorios del municipio y efectuadas clasificaciones en cuanto a la edad de los animales, seguida de las clasificaciones referentes a las alteraciones hepáticas y renales y otra para el sexo. La alteración con mayor frecuencia en la población de perros geriátricos y adultos resultó ser Fosfatasa Alcalina con 50,8\% (2.206 / 4.341) y 41,6\% (979 / 2.352) respectivamente. El segundo analito con la mayor frecuencia de alteraciones fue Urea con 44,2\% (1.923 / 4.341) en perros geriatras y 29,9\% (705/2.352) en perros mayores. La relevancia del sexo con relación a la edad se mostró significativa en los marcadores Fosfatasa Alcalina y Urea.

Palabras clave: alanina aminotransferasa, perro, creatinina, diagnóstico, fosfatasa alcalina, ancianos, urea

\section{Introdução}

Animais geriatras representam uma generosa parcela da população de pacientes atendidos na rotina veterinária, sendo estimado um aumento gradativo e significativo conforme o surgimento de novas tecnologias com o passar dos anos (Metzger, 2005; Fortney, 2012). A definição do envelhecimento consiste no acumulo de alterações continuas em um organismo que pode estar apenas associada a determinadas afecções ou pode ser o responsável pelo surgimento delas, tanto quanto o declínio de funções fisiológicas e morte (Fortney, 2004; Fortney, 2012) A idade considerada real de um organismo é chamada também de idade cronológica, devendo sempre ser diferenciada da idade biológica, que condiz com a funcionalidade de cada sistema do organismo. Ressalta-se então que animais com a mesma idade cronológica podem obter diferentes mudanças funcionais em seus organismos (Fortney, 2012). Esta relação fortemente associada ao declínio fisiológico varia entre espécies, raças, porte, doenças aos quais já 
foram expostos, estado nutricional e até mesmo entre animais da mesma ninhada (Laflamme, 2005; Fortney, 2012).

O fígado tem funções básicas que englobam desde funções secretoras até funções metabólicas e vasculares. Devido a quantidade e importância destas funções, foram desenvolvidas várias técnicas para mensurar o desempenho, bem como apontar possíveis lesões neste órgão (Guyton, 1997). Os exames laboratoriais hepáticos devem ser divididos em testes que mensuram lesão nos hepatócitos, que detectam colestase e por fim os que avaliam a função hepática (Thrall et al., 2015). Em relação às enzimas presentes no fígado, a alanina aminotransferase (ALT) é uma enzima citosólica de escape, encontrada primariamente em hepatócitos, sendo que, quando há persistência nas elevações desta enzima, é indicativa a existência de dano hepático (Metzger \& Rebar, 2012; Thrall et al., 2015). Outro marcador capaz de fornecer importantes informações quanto ao perfil hepático é a fosfatase alcalina (FA), que se trata de uma enzima de indução ligada às membranas (Watson \& Bunch, 2009). A maior parte de FA de origem sérica é hepática, tornandoa, desta forma, um marcador de muito valor na avaliação deste órgão (Gonzalez \& Silva 2006).

Os rins, por sua vez, possuem funções excretoras, no controle de concentrações e produção de hormônios (Guyton, 1997). A função renal pode ser avaliada pelas concentrações de ureia, creatinina e resíduos nitrogenados do catabolismo de proteínas no soro sanguíneo (Thrall et al., 2015). A ureia é sintetizada no fígado e excretada, como via principal, pelo rim, sendo a sua concentração no filtrado glomerular a mesma igual à concentração sanguínea (Di Bartola, 2005). A creatinina, por sua vez, é tida como produto da desidratação interna e não enzimática da creatina e da desfosforilação do fosfato de creatina no tecido muscular, tendo sua excreção de forma renal (Braun, et al., 2003). As concentrações de creatinina no soro são normalmente utilizadas para estimar a taxa de filtração glomerular e classificar a insuficiência renal crônica, sendo o marcador de função renal mais utilizada em medicina veterinária ( $\underline{\text { Cobrin et }}$ al., 2013).

As análises sanguíneas são fortes aliadas e muito usadas na medicina veterinária com o objetivo de identificar alterações causadas por conta de alguma doença, ou até mesmo para monitoramento da saúde de um animal saudável
(Comazzi et al., 2004). Para uma avaliação clínica individual, ou até mesmo para avaliar populações animais, a medicina veterinária vem utilizando extensivamente os perfis bioquímicos (Payne \& Payne, 1987). Os valores bioquímicos, se interpretados de forma correta, concedem referências quanto ao estado clínico de um animal, bem como seu equilíbrio nutricional, status imunológico, acompanhamento de tratamentos e prognósticos (González, 2001).

Por ser uma ferramenta tão importante para o diagnóstico de enfermidades, tanto em seu estágio inicial quanto avançado, se torna de extrema importância o uso da análise de bioquímica sérica em cães idosos, buscando detectar afecções de forma mais precoce possível nesta fase, onde o animal se torna tão susceptível as mesmas. Portanto, procurou-se estabelecer a ocorrência destas alterações e possíveis etiologias, sendo elas doenças ou alterações decorrentes do desgaste fisiológico.

Em suma, o objetivo deste estudo foi avaliar a ocorrência das alterações de exames bioquímicos que indicam modificações nas funções renal e hepática de cães e classificá-las em relação ao sexo e idade.

\section{Material e Métodos}

Através de um contato prévio com os laboratórios BADANIEVET e MEDIVET da cidade de Joinville - Santa Catarina, foram requeridos todos exames bioquímicos realizados nos anos de 2015 a 2017.

A primeira triagem foi para selecionar os animais da espécie canina, e em seguida para separar os exames por idade, criando dois grandes grupos, sendo o grupo 1 caracterizado pelos animais sênior (de 7 a 9 anos de idade) e o grupo 2 caracterizado pelos animais geriatras (a partir de 10 anos de idade).

Os exames selecionados anteriormente, foram separados em subgrupos dividindo-se em animais que possuem alterações bioquímicas nas concentrações dos analitos alanina aminotransferase e fosfatase alcalina, que são indicadores de alterações hepáticas, e nas concentrações dos analitos ureia e creatinina que são indicadores de alterações renais, baseando-se nos valores de referências publicados por González \& da Silva (2017). Essa triagem deu origem a oito subgrupos, sendo quatro deles pertencentes às alterações de cada analito dentro 
do grupo senior, e outros quatro as alterações dos analitos dentro do universo geriatra.

Dentro dos subgrupos, ainda foi realizada uma divisão final referente as sexos dos animais, fazendo com que cada um dos oito subgrupos possuisse uma divisão por sexo.

A análise estatística realizada dentro dos dois grandes blocos, comparando as alterações entre os grupos sêniors e geriátricos, bem como a relação entre machos e fêmea, foi através do programa $R$ pelo teste de qui-quadrado. Uma análise descritiva de todos os dados encontrados também foi efetuada.

\section{Resultados e Discussão}

No período de 2015 a 2017, os laboratórios MediVet e Badanievet obtiveram um total de 23.092 laudos bioquímicos realizados. Dentre estes, 4.341 laudos eram pertencentes a cães geriatras e 2.352 pertencentes a cães seniors.

Com os resultados obtidos neste estudo, foi possível observar que o analito bioquímico com maior prevalência de alterações foi Fosfatase Alcalina, fato que ocorreu em ambos os grupos, geriatras e seniors (Figuras 1 e 2). No estudo de Comazzi et al. (2004), foram obtidos valores semelhantes onde $39,1 \%$ dos cães apresentou esta alteração e Alef et al. (2008) com 35,9\% dos cães afetados. Já no estudo de Davies \& Kawaguchi (2014) foram relatados resultados inferiores, tendo $19,3 \%$ dos seus cães apresentado aumento da Fosfatase Alcalina. Comazzi et al. (2004) detectou aumento significativo da atividade da FA em cães idosos comparativamente a cães de meia-idade, isso se mostrou presente neste estudo onde houve diferença estatística deste analito entre os grupos de animais seniors e geriatras (Tabela 1).

Dentro das alterações hepáticas, o aumento de ALT obteve significância estatística com relação a idade (Tabela 1) e teve valores semelhantes aos de Comazi et al. (2004) em que $17,4 \%$ dos cães apresentaram aumento deste analito e por Davies \& Kawaguchi (2014) em que $14,4 \%$ dos cães tinham esta alteração. Weeb et al. (2012) detectou valores mais baixos com 9,4\% dos animais exibindo esta alteração. Alef et al (2008) obteve resultados totalmente distintos, tendo detectado valores da ALT que excediam o limite superior de referência em 43,8\% dos cães, apesar de apenas $3.8 \%$ terem sido clinicamente relevantes. Esta discrepância nos resultados pode ter como responsável as diferenças de idades e critérios de inclusão dos animais entre os estudos, já que ambos os estudos usaram valores de idade média diferentes, sendo Weeb et al. (2012) 9 anos e Alef et al (2008) 5 anos. No entanto, o fator idade não parece justificar a grande diferença existente na prevalência de alterações da ALT em relação aos restantes estudos.

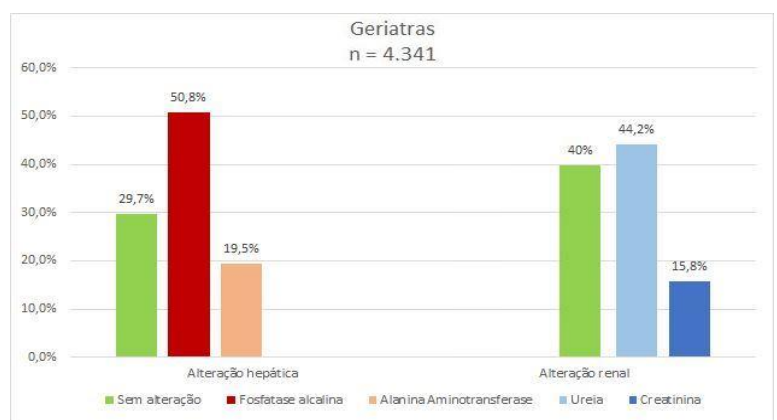

Figura 1. Caracterização da amostra referente às alterações de âmbito hepático e renal em animais geriatras

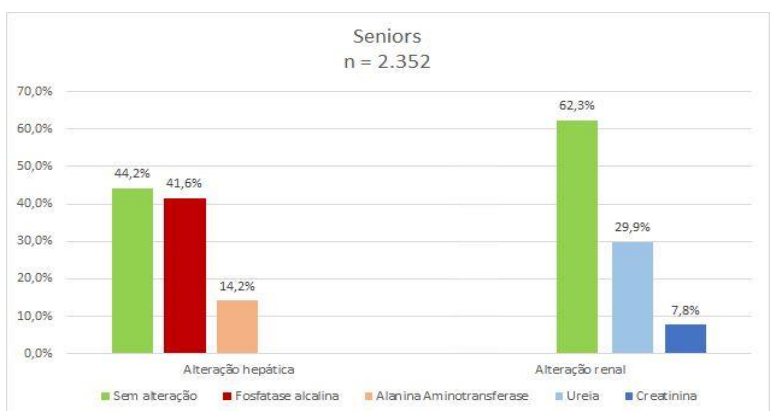

Figura 2. Caracterização da amostra referente às alterações de âmbito hepático e renal em animais seniors.

Tabela 1. Caracterização da amostra quanto as alterações dos biomarcadores e faixa etária.

\begin{tabular}{lll}
\hline Marcadores & Geriatra & Senior \\
\hline Fosfatase alcalina & $50,8 \%^{\mathrm{A}}$ & $41,6 \%{ }^{\mathrm{B}}$ \\
\hline Alanina Aminotransferase & $19,5 \%{ }^{\mathrm{A}}$ & $14,2 \%{ }^{\mathrm{B}}$ \\
\hline Ureia & $44,2 \%^{\mathrm{A}}$ & $29,9 \%^{\mathrm{B}}$ \\
\hline Creatinina & $15,8 \%{ }^{\mathrm{A}}$ & $7,8 \%{ }^{\mathrm{B}}$ \\
\hline
\end{tabular}

* Números seguidos de letras diferentes na mesma linha, diferem estatisticamente $(\mathrm{p}<0,001)$ por meio do teste de quiquadrado.

O aumento destas enzimas sugere, portanto, que o desempenho deste órgão possa estar sendo prejudicado. Segundo Gilon \& Graves (2011), $70 \%$ dos cães com idade igual ou superior a 14 anos têm hiperplasia nodular hepática benigna. Também Cooper \& Webster (2006) referem que a hiperplasia nodular é uma causa comum de aumentos leves a moderados nas enzimas hepáticas em cães assintomáticos com idade acima de 8 anos. Outras causas de aumento de ALT podem ser levantadas, tais como a diminuição do fluxo sanguíneo e da taxa de regeneração hepática secundariamente ao envelhecimento (Baetge \& 
Matthews 2012; Hoskins 2004). Demais autores como Joubert (2007) e Hughes (2008) referem ainda a existência de uma queda na capacidade de desintoxicação do fígado com aumento da idade, enquanto que Cooper \& Webster (2006) consideraram que a lipidose hepática e a hiperplasia nodular hepática são as alterações mais comuns do fígado do cão velho. Ressalta-se também as neoplasias hepáticas primárias ou metastáticas como fatores importantes a se levar em consideração quando o assunto é cães idosos com alterações das enzimas hepáticas (Twedt; 2009).

Os resultados encontrados referentes a função renal demonstraram que o analito ureia foi o segundo com maior frequência de alterações no presente estudo em ambos os grupos etários (Figuras 1 e 2). Nos estudos realizados por Comazzi et al. (2004) e Alef et al. (2008), os resultados foram mais próximos aos valores encontrados no grupo de seniors, tendo em vista que se verificou aumento da ureia em $15,5 \%$ e $16,7 \%$ dos animais, respectivamente. Já Davies \& Kawaguchi (2014) obtiveram resultados muito inferiores se comparados a ambos os grupos seniors e geriatras, com $9,1 \%$ de alterações de ureia em cães. Foi possível verificar que existe significância estatística entre o marcador ureia e idade (Tabela 1), sugerindo-se que à medida que a idade aumenta a ureia tem também tendência a aumentar. Alef et al. (2008) não encontrou relações significativas entre a idade e a ureia. Entretanto, Fukuda et al. (2012) também verificou aumento da ureia com envelhecimento. Da mesma maneira Comazzi et al. (2004) encontraram valores significativamente maiores em animais geriátricos do que em animais de meia-idade. Medaille et al., (2004), Grauer (2005) e Mugford et al. (2013) declararam o efeito das dietas ricas em proteínas podendo causar aumentos moderados da ureia em animais saudáveis, sendo recomendado um jejum de 8 a 12 horas.

No que diz respeito a creatinina, esta obteve menos alterações do que a ureia em ambos os grupos etários (Figuras 1 e 2), demonstrando diferença estatística quanto a idade (Tabela 1). Outros autores obtiveram resultados semelhantes ao grupo de cães seniors como foi o caso de Comazzi et al. (2004), Alef et al. (2008) e Davies e Kawaguchi (2014) que verificaram que $8.1 \%$, $5.9 \%$ e $4.2 \%$ dos seus cães tinham aumento deste parâmetro, respectivamente. Existem situações não patológicas que causam alterações notáveis quanto a creatinina. Segundo Braun et al. (2003) e Medaille (2004), nas primeiras horas após a alimentação com carne, a creatinina pode aumentar e permanecer elevada até 12 horas. Braun et al. (2003) refere ainda que a desidratação é uma explicação possível para o aumento desta bioquímica, assim como o exercício físico intenso.

Existem ocasiões onde as alterações marcadas destes analitos podem vir a dar indícios de uma saúde renal prejudicada. A diminuição da taxa de filtração glomerular secundária ao envelhecimento, também poderia explicar o aumento da creatinina (Hughes 2008) Da mesma forma, as hemorragias gastrointestinais provocam o aumento da ureia, pois o sangue representa uma fonte de proteína endógena (Medaille et al.2004; Mugford et al. 2013). Devem ser consideradas também algumas doenças responsáveis pelo aumento do catabolismo, como febre, fome e infecção (Grauer 2005). Além disso, deve ser avaliado o grau de desidratação, choque e doença cardiovascular como causadores de diminuição da perfusão renal e consequentemente da diminuição da taxa de filtração glomerular levando ao aumento de ureia e creatinina (Grauer 2005; Mugford et al. 2013).

A comparação das variáveis quanto aos dois gêneros demonstrou, que todos os marcadores bioquímicos foram maiores em fêmeas comparativamente a machos, tanto na população amostral sênior (Figuras 4 e 6 ) quanto geriatra (Figuras 3 e $\underline{5}$ ). Entretanto, de acordo com a tabela $\underline{2}$, apenas fosfatase alcalina e ureia apresentaram uma distribuição significativa.

Tabela 2 - Caracterização da amostra quanto as alterações dos biomarcadores, faixa etária e gênero.

\begin{tabular}{|c|c|c|c|c|}
\hline Marcadores & Fêmeas Geriatras & Machos Geriatras & Fêmeas Seniors & Machos Seniors \\
\hline Fosfatase alcalina & $63,8 \%{ }^{\mathrm{A}}$ & $36,2 \%{ }^{\text {в }}$ & $60 \%{ }^{A}$ & $40 \%^{\mathrm{B}}$ \\
\hline Alanina Aminotransferase & $58,3 \%$ A & $41,7 \% \mathrm{~A}$ & $57,8 \%$ A & $41,2 \% \mathrm{~A}$ \\
\hline Ureia & $60,5 \%{ }^{\mathrm{A}}$ & $39,5 \%$ в & $55,6 \%{ }^{\mathrm{A}}$ & $44,5 \%$ в \\
\hline Creatinina & $61,8 \% \mathrm{~A}$ & $38,2 \% \mathrm{~A}$ & $56 \%{ }^{\mathrm{A}}$ & $44 \%{ }^{\mathrm{A}}$ \\
\hline
\end{tabular}

* Números seguidos de letras diferentes na mesma linha, diferem estatisticamente $(p<0,05)$ por meio do teste de qui-quadrado. 
Apesar de terem sido encontrados valores significativos referentes a ureia no presente estudo, Misbach et al. (2014) não observaram nenhum efeito do gênero nos valores de ureia em cães. Estudos acerca da relação do gênero com as alterações bioquímicas de função hepática e renal estão presentes em pequeno número no meio científico, o que sugere que mais estudos deverão ser realizados sobre o assunto.

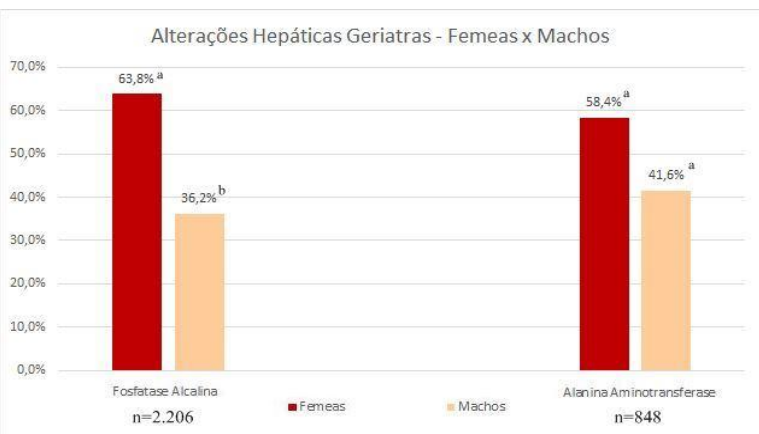

Figura 3. Caracterização da amostra referente às alterações de âmbito hepático em animais geriatras fêmeas e machos. *Números seguidos de letras diferentes no mesmo analito, diferem estatisticamente $(\mathrm{p}<0,05)$ por meio do teste de quiquadrado.

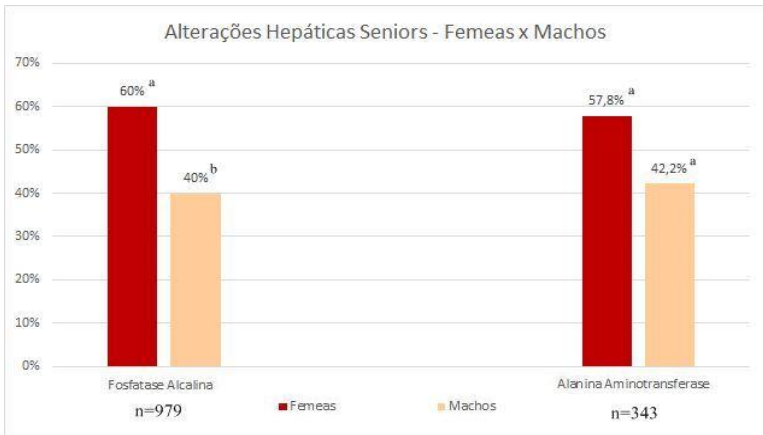

Figura 4. Caracterização da amostra referente às alterações de âmbito hepático em animais seniors fêmeas e machos. *Números seguidos de letras diferentes no mesmo analito, diferem estatisticamente $(\mathrm{p}<0,05)$ por meio do teste de quiquadrado.

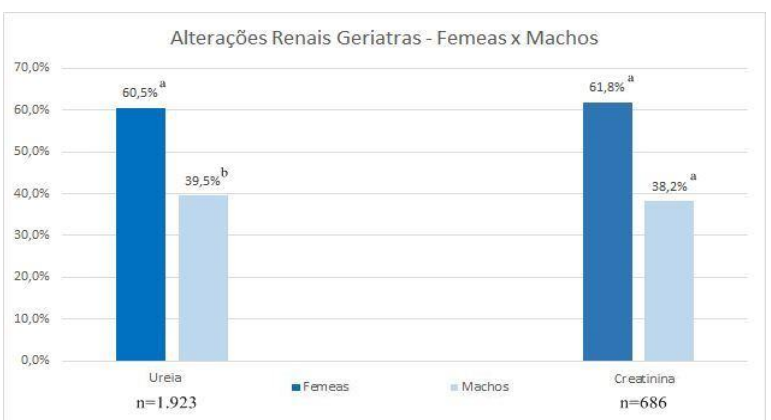

Figura 5. Caracterização da amostra referente às alterações de âmbito renal em animais geriatras fêmeas e machos.

* Números seguidos de letras diferentes no mesmo analito, diferem estatisticamente $(\mathrm{p}<0,05)$ por meio do teste de quiquadrado.

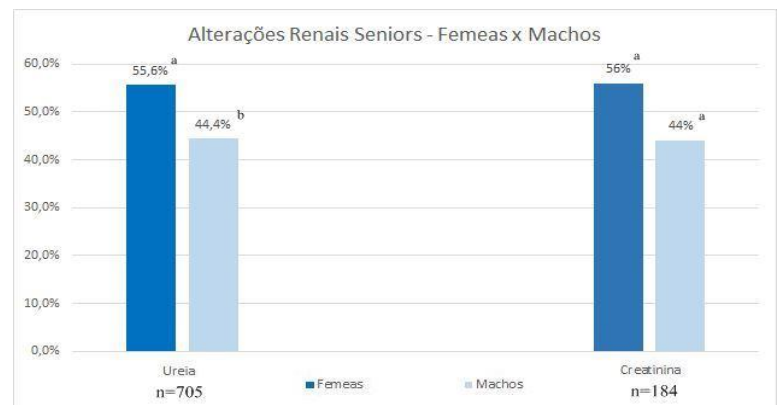

Figura 6. Caracterização da amostra referente às alterações de âmbito renal em animais seniors fêmeas e machos.

*Números seguidos de letras diferentes no mesmo analito, diferem estatisticamente $(\mathrm{p}<0,05)$ por meio do teste de quiquadrado.

\section{Conclusão}

Através dos resultados obtidos, sugere-se que talvez possa haver uma influência da idade na funcionalidade hepática e renal de cães uma vez que, a quantidade de alterações foi maior em cães geriatras que seniors em todos os analitos estudados. Indica-se também, que cães geriatras podem estar mais propensos a desenvolver mais alterações hepáticas do que alterações renais. Quanto à influência do sexo, os resultados sugerem que fêmeas possam desenvolver alterações com maiores frequências que machos nos analitos fosfatase alcalina e ureia, apesar da presença de estudos contraditórios.

Desta forma, o presente estudo torna possível, dentro dos limites especulativos, traçar um perfil acerca das alterações, fisiológicas ou não, referentes à idade de cães em suas funcionalidades hepáticas e renais.

\section{Referências bibliográficas}

Alef, M., von Praun, F. \& Oechtering, G. 2008. Is routine pre-anaesthetic haematological and biochemical screening justified in dogs? Veterinary Anesthesia and Analgesia, 35, 132140.

Baetge, C. L. \& Matthews, N. S. 2012. Anesthesia and Analgesia for Geriatric Veterinary Patients. Veterinary Clinics of North America: Small Animal Practice, 42, 643-653.

Braun, J. P., Lefebvre, H. P. \& Watson, A.D. J. 2003. Creatinine in the dog: a review. Veterinary Clinic Pathology, 32, 162-172.

Cobrin, A. R., Blois, S. L., Kruth, S. A., AbramsOgg, A. C. G. \& Dewey, C. 2013. Biomarkers in the assessment of acute and chronic kidney diseases in the dog and cat. Journal of Small Animal Practice, 54, 647-655. 
Comazzi, S., Pieralisi, C. \& Bertazzolo, W. 2004. Haematological and biochemical abnormalities in canine blood: frequency and associations in 1022 samples. Journal of Small Animal Practice, 45, 343-349.

Cooper, J., \& Webster, C. R. L. 2006. The diagnostic approach to asymptomatic dogs with elevated liver enzymes activities. veterinarymedicine.dvm360, 1-7.

Davies, M., Kawaguchi, S. 2014. Pregeneral anaesthetic blood screening of dogs and cats attending a UK practice. Veterinary Record, 174.

DiBartola, S. P. 2005. Chapter 257 - Renal Disease: Clinical Approach and Laboratory Evaluation. In: Ettinger,S., Feldman, E. (Eds) Textbook of Veterinary Internal Medicine Volume II, 6 ${ }^{\mathrm{a}} \mathrm{Ed} ., \mathrm{p}$ 1716-1730, Elsevier Saunders, USA.

Fortney, W. 2004. Chapter 1 - Geriatrics and Aging. In: Hoskins, J. (Eds) Geriatric and Gerontology of the dog and cat. $2^{\mathrm{a}}$ Ed., p 1-4, Sauders, USA.

Fortney, W. 2012. Implementing a successful senior/geriatric health care program for veterinarians, veterinary technicians and office managers. Veterinary Clinics of North America: Small Animal Practice, 42, 823-834.

Fukuda, H. Mori, A., Urumuhan, N., Lee, P., Oda, H., Saeki, K, Kurishima, M., Nozawa, S., Mizutani, H., Mishina, S. Arai, T. \& Sako, T. 2012. Characterization and Comparison of Insulin Resistance Induced by Cushing Syndrome or Diestrus against Healthy Control Dogs as Determined by Euglycemic Hyperinsulinemic Glucose Clamp Profile Glucose Infusion Rate Using an Artificial Pancreas Apparatus. Journal of Veterinary Medical Science, 74 (11), 1527-1530.

Gilon, C., Graves, T. 2011. Interpretation of laboratory tests for canine Cushing's Syndrome. Topics in Companion Animal Medicine, 26, 98-108.

Gonzalez, F. H. D., Carvalho, V., Möller, V. A. \& Duarte, F. R. 2001. Perfil bioquímico sanguíneo de cães e gatos na cidade de Porto Alegre, Rio Grande do Sul, Brasil. Acta Scientiae Veterinariae, 29, p. 1-6.

Gonzalez, F. H. D \& Silva S. C. 2006. Introdução à bioquímica clínica veterinária. $2^{\mathrm{a}}$ ed.,
Editora da Universidade Federal do Rio Grande do Sul, Porto Alegre.

González, F. H. D. \& da Silva, S. C. 2017. Introdução à bioquímica clínica Veterinária. $3^{\mathrm{a}}$. Edição, Editora UFRGS, Porto Alegre, $578 \mathrm{p}$.

Grauer, G. F. 2005. Early Detection of Renal Damage and Disease in Dogs and Cats. Veterinary Clinics of North America: Small Animal Practice, 35, 581-596.

Guyton Arthur C. 1997. Tratado de Fisiologia Médica, 9 ed., Guanabara Koogan, Rio de Janeiro, Brasil.

Hoskins, J. 2004. Chapter 13 - The Liver rand Exocrine Pancreas. In: Hoskins, J. (Eds) Geriatric and Gerontology of the dog and cat. $2^{\mathrm{a}}$ Ed., Sauders, USA.

Hughes, J. M. L. 2008. Anaesthesia for the geriatric dog and cat. Irish Veterinary Journal, 61, 380-387.

Joubert, K. E. 2007. Pre-anaesthetic screening of geriatric dogs. Journal of South African Veterinary Association, 78, 31-35.

Laflamme, D. P. 2005. Nutrition for Aging Cats and Dogs and the Importance of Body Condition. Veterinary Clinics of North America: Small Animal Practice, 35, 713-742.

Medaille, C., Trumel, C., Concordet, D., Vergez., F. \& Braun, J. P. 2004. Comparison of Plasma/Serum Urea and Creatinine Concentrations in the Dog: A 5-Year Retrospective Study in a Commercial Veterinary Clinical Pathology Laboratory. Journal of Veterinary Medicine. A, Physiology Pathology Clinical Medicine, 51, 119-123.

Metzger, F. L. 2005. Senior and Geriatric Care Programs for Veterinarians. Veterinary Clinics of North America: Small Animal Practice, 35, 743-753.

Metzger, F. L. \& Rebar, A. H. 2012. Clinical pathology interpretation in geriatric veterinary patients. Veterinary Clinics of North America: Small Animal Practice, 42, 615-629.

Misbach, C., Chetboul, V., Concordet, D., Médaille, C., Gruet, C., Speranza, S., Hoffmann, A.C., Rocha, A., Balouka, D., Petit, A. M. P., Trehiou-Sechim, E., Pouchelon, J. \& Lefebvre, H. P. 2014. Basal plasma concentrations of routine variables and packed cell volume in clinically healthy adult 
small- sized dogs: effect of breed, body weight, age and gender and establishment of reference intervals. Veterinary Clinic Pathology, 43, 371-380.

Mugford, A., Li., R., Humm, K. 2013. Acute Kidney injury in dogs and cats 1 . pathogenesis and diagnosis. In Practice, 35, 253-264.

Payne, J. M., Payne, S. 1987. The Metabolic Profile Test. Oxford University Press, New York, p. 179.

Thrall M. A, Weiser, G., Allison Robin, W. \& Campbell Terry, W. 2015. Hematologia e bioquímica clínica veterinária, 2 ed., Guanabara Koogan, Rio de Janeiro.

Twedt, D. C. 2009. Abnormal liver enzymes: a practical approach. Delaware Valley Academy of Veterinary Medicine, 1-5.
Watson, P. J., Bunch, S. E. 2009. Capitulo 36 Testes Diagnósticos para o Sistema Hepatobiliar. In: Nelson, C., Couto, G., (Eds) Medicina Interna de Pequenos Animais, $4^{\mathrm{a}} \mathrm{Ed}$, Mosby Elsevier, USA.

Webb, J., Kirby, G., Nykamp, S. \& Gauthier, M. 2012. Ultrasonografic and laboratory screening in clinically normal mature golden retriever dogs. Canadian Veterinary Journal, 53, 626630 .

Recebido: 16 Julho, 2018

Aprovado: 23 Agosto, 2018

Publicado: 17 Setembro, 2018

Licenciamento: Este artigo é publicado na modalidade Acesso Aberto sob a licença Creative Commons Atribuição 4.0 (CC-BY 4.0), a qual permite uso irrestrito, distribuição, reprodução em qualquer meio, desde que o autor e a fonte sejam devidamente creditados. 